Yesheng Li, Huating Chen, Han Chen, Xingen Hu and Hongping Xiao*

\title{
The crystal structure of diaqua-bis $\left(\mu_{2}-3-((3-a c e t y l-5\right.$-carboxyphenyl) oxidophosphoryl)-5-carboxybenzoato- $\left.\mathrm{K}^{2} \mathrm{O}: \mathrm{O}^{\prime}\right)$ bis(5,5'-dimethyl-2,2'- bipyridine-k $\left.{ }^{2} N, N^{\prime}\right)$ zinc(II), $\mathrm{C}_{56} \mathrm{H}_{46} \mathrm{~N}_{4} \mathrm{O}_{22} \mathrm{P}_{2} \mathrm{Zn}_{2}$
}

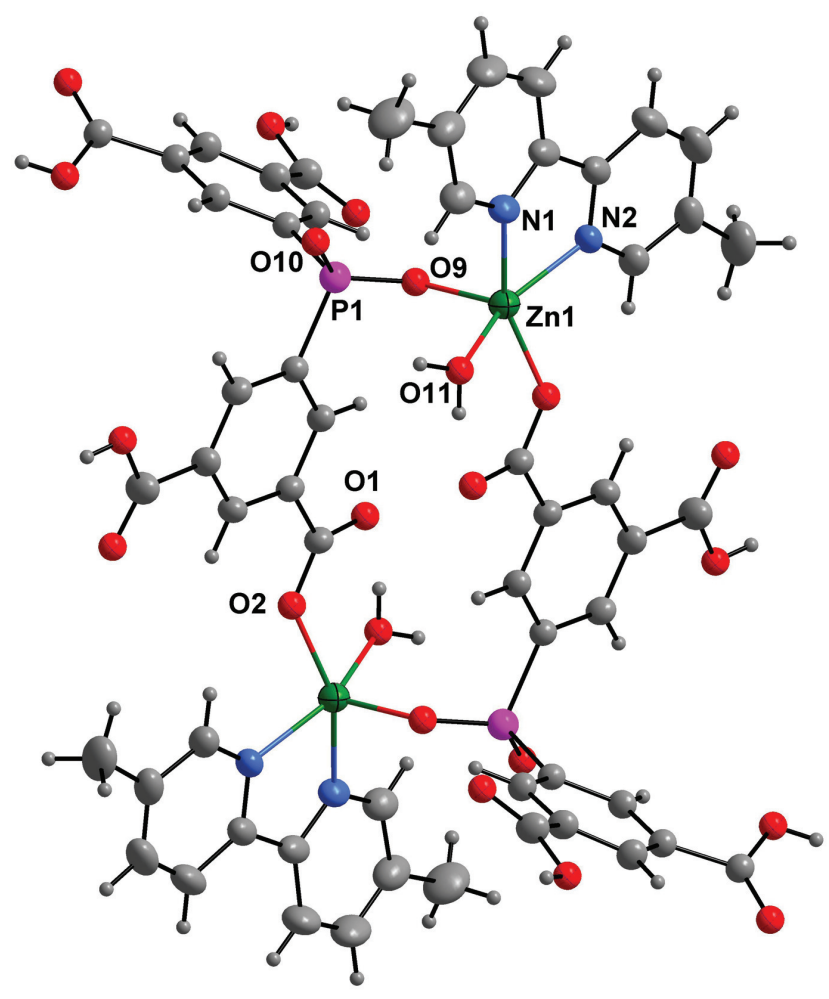

https://doi.org/10.1515/ncrs-2019-0517

Received July 19, 2019; accepted September 22, 2019; available online October 9, 2019

\section{Abstract \\ $\mathrm{C}_{56} \mathrm{H}_{46} \mathrm{~N}_{4} \mathrm{O}_{22} \mathrm{P}_{2} \mathrm{Zn}_{2}, \quad$ monoclinic, $P 2_{1} / c$ (no. 14), $a=$ 9.6537(4) $\AA, \quad b=31.1374(11) \AA, \quad c=9.8927(4) \AA$, $\beta=112.709(2)^{\circ}, \quad V=2743.13(19) \AA^{3}, \quad Z=2, \quad R_{\mathrm{gt}}(F)=0.0341$, $w R_{\text {ref }}\left(F^{2}\right)=0.0934, T=296(2) \mathrm{K}$.}

CCDC no.: 1955108
The dinuclear title structure is shown in the figure. Table 1 contains crystallographic data and Table 2 contains the list of the atoms including atomic coordinates and displacement parameters.

Table 1: Data collection and handling.

\begin{tabular}{ll}
\hline Crystal: & Colorless prism \\
Size: & $0.26 \times 0.22 \times 0.21 \mathrm{~mm}$ \\
Wavelength: & Cu $K \alpha$ radiation $(1.54178 \AA$ A $)$ \\
$\mu:$ & $2.39 \mathrm{~mm}^{-1}$ \\
Diffractometer, scan mode: & Bruker APEX-II, $\varphi$ and $\omega$ \\
$\theta_{\text {max }}$, completeness: & $65.5^{\circ}, 97 \%$ \\
$N(h k l)_{\text {measured }}, N(h k l)_{\text {unique }}, R_{\text {int }}:$ & $11995,4601,0.014$ \\
Criterion for $I_{\text {obs }}, N(h k l)_{\text {gt }}:$ & $I_{\text {obs }}>2 \sigma\left(I_{\text {obs }}\right), 4464$ \\
$N(\text { param })_{\text {refined }}:$ & 393 \\
Programs: & Bruker [1], Diamond [2], SHELX \\
& {$[3,4]$} \\
\hline
\end{tabular}

\section{Source of material}

A mixture of $\mathrm{ZnSO}_{4} \cdot 7 \mathrm{H}_{2} \mathrm{O}$ (0.0386 g), tri(3,5-dicarboxylic phenyl)phosphine oxide $\quad(0.0249 \mathrm{~g}), \quad 5,5^{\prime}$-methyl-2,2'bipyridine $(0.0153 \mathrm{~g})$ and water $(10 \mathrm{~mL})$ was sealed in a $15 \mathrm{~mL}$ Teflon-lined steel autoclave and heated at $423 \mathrm{~K}$ for three days and then cooled to room temperature. Colorless block crystals were obtained (yield ca. 37\% based on phosphine). The product is insoluble in water and common organic solvents. FT-IR $\left(\mathrm{KBr}, \mathrm{cm}^{-1}\right)$ : $3450 \mathrm{w}, 1714 \mathrm{~s}, 1692 \mathrm{~m}, 1597 \mathrm{~m}, 1544 \mathrm{~m}$, 1509 m, 1409 s, 1331 m, 1279 s, 1234 m, 1129 m, 1059 m, 824 m, $753 \mathrm{~m}, 687 \mathrm{~m}$.

\section{Experimental details}

The $\mathrm{H}$ atoms were positioned geometrically and allowed to ride on their parent atoms at distances of $\quad \mathrm{Csp}^{2}-\mathrm{H}=0.93 \AA \quad$ with $\quad U_{\text {iso }}(\mathrm{H})=1.2 U_{\text {eq }}(\mathrm{C}), \quad \mathrm{Csp}^{3}$ $\mathrm{H}=0.96 \AA$ with $U_{\text {iso }}(\mathrm{H})=1.5 U_{\text {eq }}(\mathrm{C})$ and $\mathrm{O}-\mathrm{H}=0.85 \AA$ with $U_{\text {iso }}(\mathrm{H})=1.5 U_{\text {eq }}(\mathrm{O})$, respectively.

\section{Comment}

The design and construction of coordination polymers are attracting interest due to their specific structures and potential applications [5-7]. In order to achieve these goals, it is necessary to select suitable ligands like phosphine oxides [8-10]. For example, in our previous works, a series of 
Table 2: Fractional atomic coordinates and isotropic or equivalent isotropic displacement parameters $\left(\AA^{2}\right)$.

\begin{tabular}{|c|c|c|c|c|}
\hline Atom & $x$ & $y$ & $z$ & $U_{\text {iso }} * / U_{\text {eq }}$ \\
\hline Zn1 & $0.56539(3)$ & $0.05904(2)$ & $0.41400(3)$ & $0.03230(11)$ \\
\hline P1 & $0.55669(5)$ & $0.13201(2)$ & $0.18781(5)$ & $0.02594(13)$ \\
\hline 01 & $0.62563(19)$ & $0.00431(6)$ & $-0.13473(19)$ & $0.0504(4)$ \\
\hline 02 & $0.41927(18)$ & $0.00032(5)$ & $-0.33994(17)$ & $0.0436(4)$ \\
\hline 03 & $0.0381(2)$ & $0.11430(8)$ & $-0.4553(2)$ & $0.0781(7)$ \\
\hline 04 & $0.0456(2)$ & $0.16065(6)$ & $-0.2826(2)$ & $0.0573(5)$ \\
\hline $\mathrm{H} 4$ & -0.030432 & 0.169954 & -0.347643 & $0.086^{\star}$ \\
\hline 05 & $0.56429(16)$ & $0.27494(5)$ & $-0.09109(17)$ & $0.0404(4)$ \\
\hline H5 & 0.542523 & 0.298041 & -0.133937 & $0.061^{\star}$ \\
\hline 06 & $0.79124(19)$ & $0.30468(5)$ & $0.0047(2)$ & $0.0522(5)$ \\
\hline 07 & $1.18952(17)$ & $0.18832(6)$ & $0.1758(2)$ & $0.0514(4)$ \\
\hline $\mathrm{H} 7$ & 1.271495 & 0.176999 & 0.194215 & $0.077^{\star}$ \\
\hline 08 & $1.11941(18)$ & $0.12134(6)$ & $0.1963(2)$ & $0.0564(5)$ \\
\hline 09 & $0.63644(16)$ & $0.09667(5)$ & $0.29121(15)$ & $0.0332(3)$ \\
\hline 010 & $0.45649(15)$ & $0.16175(5)$ & $0.23122(16)$ & $0.0328(3)$ \\
\hline 011 & $0.33616(18)$ & $0.05716(5)$ & $0.2936(2)$ & $0.0459(4)$ \\
\hline $\mathrm{H} 11 \mathrm{~A}$ & 0.328442 & 0.037761 & 0.230658 & 0.069 * \\
\hline H11B & 0.276688 & 0.077719 & 0.253040 & $0.069^{\star}$ \\
\hline N1 & $0.5413(2)$ & $0.10551(6)$ & $0.5605(2)$ & $0.0394(4)$ \\
\hline N2 & $0.7580(2)$ & $0.04855(6)$ & $0.6040(2)$ & $0.0397(4)$ \\
\hline $\mathrm{C} 1$ & $0.4490(2)$ & $0.10918(6)$ & $0.0100(2)$ & $0.0292(4)$ \\
\hline $\mathrm{C} 2$ & $0.5065(2)$ & $0.07334(7)$ & $-0.0337(2)$ & $0.0317(4)$ \\
\hline $\mathrm{H} 2$ & 0.595561 & 0.061152 & 0.030397 & $0.038^{\star}$ \\
\hline C3 & $0.4332(2)$ & $0.05536(6)$ & $-0.1716(2)$ & $0.0324(4)$ \\
\hline $\mathrm{C} 4$ & $0.2998(2)$ & $0.07372(7)$ & $-0.2657(2)$ & $0.0359(5)$ \\
\hline $\mathrm{H} 4 \mathrm{~A}$ & 0.249685 & 0.061925 & -0.3 & $0.043^{\star}$ \\
\hline C5 & $0.2402(2)$ & $0.10934(7)$ & $-0.2238(2)$ & $0.0358(5)$ \\
\hline C6 & $0.3149(2)$ & $0.12736(7)$ & $-0.0851(2)$ & $0.0336(4)$ \\
\hline H6 & 0.275423 & 0.151311 & -0.056535 & 0.040 * \\
\hline $\mathrm{C} 7$ & $0.4995(2)$ & $0.01679(7)$ & $-0.2173(2)$ & $0.0348(5)$ \\
\hline $\mathrm{C} 8$ & $0.0982(3)$ & $0.12776(8)$ & -0.3 & $0.0470(6)$ \\
\hline $\mathrm{C} 9$ & $0.6958(2)$ & $0.16556(6)$ & $0.1588(2)$ & $0.0280(4)$ \\
\hline C10 & $0.6537(2)$ & $0.20610(7)$ & $0.0967(2)$ & $0.0305(4)$ \\
\hline $\mathrm{H} 10$ & 0.555763 & 0.215553 & 0.073252 & $0.037^{\star}$ \\
\hline C11 & $0.7557(2)$ & $0.23263(7)$ & $0.0692(2)$ & $0.0319(4)$ \\
\hline C12 & $0.9020(2)$ & $0.21843(7)$ & $0.1032(2)$ & $0.0352(5)$ \\
\hline $\mathrm{H} 12$ & 0.971957 & 0.236359 & 0.088488 & $0.042^{*}$ \\
\hline C13 & $0.9431(2)$ & $0.17740(7)$ & $0.1591(2)$ & $0.0342(4)$ \\
\hline C14 & $0.8410(2)$ & $0.15118(7)$ & $0.1891(2)$ & $0.0326(4)$ \\
\hline H14 & 0.870287 & 0.124049 & 0.229323 & 0.039 * \\
\hline C15 & $0.7077(2)$ & $0.27465(7)$ & $-0.0061(2)$ & $0.0336(4)$ \\
\hline C16 & $1.0931(2)$ & $0.15930(7)$ & $0.1801(3)$ & $0.0379(5)$ \\
\hline C17 & $0.4295(3)$ & $0.13402(8)$ & $0.5280(3)$ & $0.0465(6)$ \\
\hline $\mathrm{H} 17$ & 0.343459 & 0.129308 & 0.444390 & $0.056^{\star}$ \\
\hline C18 & $0.4343(3)$ & $0.17020(9)$ & $0.6115(3)$ & $0.0539(7)$ \\
\hline C19 & $0.5634(4)$ & $0.17554(10)$ & $0.7349(3)$ & $0.0671(8)$ \\
\hline H19 & 0.573315 & 0.199540 & 0.793852 & $0.081^{\star}$ \\
\hline C20 & $0.6776(4)$ & $0.14602(10)$ & $0.7723(3)$ & $0.0639(8)$ \\
\hline $\mathrm{H} 2 \mathrm{O}$ & 0.763297 & 0.149616 & 0.857105 & $0.077^{\star}$ \\
\hline $\mathrm{C} 21$ & $0.6642(3)$ & $0.11076(8)$ & $0.6824(2)$ & $0.0439(5)$ \\
\hline $\mathrm{C} 22$ & $0.7803(3)$ & $0.07696(8)$ & $0.7127(2)$ & $0.0434(5)$ \\
\hline $\mathrm{C} 23$ & $0.9044(3)$ & $0.07341(10)$ & $0.8433(3)$ & $0.0578(7)$ \\
\hline $\mathrm{H} 23$ & 0.919800 & 0.093130 & 0.918321 & $0.069^{\star}$ \\
\hline C24 & $1.0051(3)$ & $0.04031(10)$ & $0.8609(3)$ & $0.0616(8)$ \\
\hline $\mathrm{H} 24$ & 1.088915 & 0.037895 & 0.947982 & $0.074^{*}$ \\
\hline $\mathrm{C} 25$ & $0.9825(3)$ & $0.01080(9)$ & $0.7505(3)$ & $0.0516(6)$ \\
\hline
\end{tabular}

Table 2 (continued)

\begin{tabular}{lrrrr}
\hline Atom & $\boldsymbol{x}$ & $\boldsymbol{y}$ & $\boldsymbol{z}$ & $\boldsymbol{U}_{\text {iso }} \boldsymbol{U}_{\text {eq }}$ \\
\hline C26 & $0.8561(3)$ & $0.01660(8)$ & $0.6233(3)$ & $0.0459(6)$ \\
H26 & 0.838569 & -0.002825 & 0.547035 & $0.055^{*}$ \\
C27 & $0.3081(4)$ & $0.20210(10)$ & $0.5669(4)$ & $0.0733(9)$ \\
H27A & 0.300754 & 0.214393 & 0.652923 & $0.110^{*}$ \\
H27B & 0.215612 & 0.187914 & 0.509981 & $0.110^{*}$ \\
H27C & 0.327221 & 0.224422 & 0.509441 & $0.110^{*}$ \\
C28 & $1.0875(3)$ & $-0.02632(11)$ & $0.7644(4)$ & $0.0699(9)$ \\
H28A & 1.186138 & -0.015541 & 0.782063 & $0.105^{*}$ \\
H28B & 1.051706 & -0.042747 & 0.675433 & $0.105^{\star}$ \\
H28C & 1.091485 & -0.044289 & 0.844712 & $0.105^{*}$ \\
\hline
\end{tabular}

coordination complexes have been obtained by (polycarboxylic phenyl)hypophosphite ligand [11-13]. Herein, we reported a new binuclear complex $\left[\mathrm{Zn}_{2}\left(\mathrm{H}_{3} \mathrm{~L}\right)_{2}(\text { bipy })_{2}\left(\mathrm{H}_{2} \mathrm{O}\right)_{2}\right]$ $\left(\mathrm{H}_{3} \mathrm{~L}=3\right.$-((3-acetyl-5-carboxyphenyl)oxidophosphoryl)-5carboxybenzoate, bipy $=5,5^{\prime}$-dimethyl-2,2'-bipyridine).

In the title complex, each $\mathrm{Zn}$ (II) is five-coordinated, with two $\mathrm{N}$ atoms from one bipy ligand, and three $\mathrm{O}$ atoms from carboxyl 02, phosphoryl 09 and aqua 011, respectively. The Zn1-N1 and Zn1-N2 bond lengths are 2.1230(18) $\AA$ and 2.0973(18) $\AA$. The $\mathrm{Zn}-\mathrm{O}$ bond lengths are in the range 1.9904(14) - 2.0698(16) A, which are found within normal ranges bond distances in the reported $\mathrm{Zn}(\mathrm{II})$ complexes [11]. The $02-Z n 1-N 1^{\mathrm{i}}$ (symmetry code: $-x+1,-y,-z$ ) and $011-$ $\mathrm{Zn} 1-\mathrm{N} 2$ bond angles are $156.39(7)^{\circ}$ and $153.16(8)^{\circ}$ and other angles are in the range $77.70(8)-104.10(7)^{\circ}$, forming a distorted square-pyramidal coordination configuration. Each $\mathrm{H}_{3} \mathrm{~L}$ anion ligand functions as $\mu_{2}$-bridge to link two different $\mathrm{Zn}$ (II) ions, forming a binuclear structure.

It is worthy noting that the tri(3,5-dicarboxyphenyl) phosphine oxidem ligand changed into (3,5-dicarboxylic phenyl)(5-carboxylic-3-carboxyphenyl) hypophosphite ligand in the hydrothermal in-situ sythesis. Moreover, the stability of the solid state structure of the title complex is enhanced by $\mathrm{O}-\mathrm{H} \cdots \mathrm{O}$ and $\mathrm{C}-\mathrm{H} \cdots \mathrm{O}$ hydrogen bonding interactions. These hydrogen bonding interactions link neighbouring binuclear units into a three-dimensional architecture.

Acknowledgements: We gratefully acknowledge financial support provided by the National Natural Science Foundation of China (grant numbers 21571144 and 21573161).

\section{References}

1. BRUKER. SAINT, APEX2 and SADABS. Bruker AXS Inc., Madison, WI, USA (2009). 
2. Brandenburg, K.: DIAMOND. Visual Crystal Structure Information System. Ver. 4.0. Crystal Impact, Bonn, Germany (2015).

3. Sheldrick, G. M.: SHELXT - integrated space-group and crystalstructure determination. Acta Crystallogr. A71 (2015) 3-8.

4. Sheldrick, G. M.: Crystal structure refinement with SHELXL. Acta Crystallogr. C71 (2015) 3-8.

5. Liu, B.; Liu, Q.; Xiao, H.; Zhang, W.; Tao, R.: Family of dumbbell $\mathrm{Ni}_{4} \mathrm{Ln}_{2}(\mathrm{Ln}=\mathrm{Pr}, \mathrm{Sm}, \mathrm{Eu}, \mathrm{Gd}, \mathrm{Tb}, \mathrm{Ho}, \mathrm{Er})$ complexes: syntheses, structures, luminescent and magnetic properties. Dalton Trans. 42 (2013) 5047-5056.

6. Li, A.; Li, L.; Song, L.; Wang, Z. H.; Chen, Q.; Yang, T.; Zhou, X. H.; Xiao, H. P.; Yin, X. J.: Guest-induced reversible structural transitions and concomitant on/off luminescence switching of an Eu(III) metal-organic framework and its application in detectin picric acid. New J. Chem. 39 (2015) 2289-2295.

7. Li, X. L.; Hu, M.; Yin, Z.; Zhu, C.; Liu, C. M.; Xiao, H. P.; Fang, S.: Enhanced single-ion magnetic and ferroelectric properties of mononuclear Dy(III) enantionmeric pairs through the coordination role of chiral ligands. Chem. Commun. 53 (2017) 3998-4001.

8. Humphrey, S. M.; Ounoulian, S. E.; Yoon, J. W.; Hwang, Y. K.; Wise, E. R.; Chang, J. S.: Hysteretic sorption of light gases by a porous metal-organic framework containing tris(paracarboxylated triphenylphosphine oxide. Chem. Commun. (2008) 2891-2893.
9. Humphrey, S. M.; Allan, P. K.; Oungoulian, S. E.; Ironside, M. S.; Wise, E. R.: Metal-organophosphine and metal-organophosphonium frameworks with layered honeycomb-like structures. Dalton Trans. (2009) 2298-2305.

10. Bohnsack, A. M.; Ibarra, I. A.; Hatfield, P. W.; Yoon, J. W.; Hwang, Y. K.; Chang, J. S.; Humphrey, S. M.: High capacity $\mathrm{CO}_{2}$ adsorption in a $\mathrm{Mg}$ (II)-based phosphine oxide coordination material. Chem. Commun. 47 (2011) 4899-4901.

11. Mo, J. P.; Hashemi, L.; He, J. L.; Feng, W. L.; Yin, Y.; Zhang, W. B.; Li, X. H.; Xiao, H. P.; Morsali, A.: Crystal structure, thermal stability and photoluminesence properties of five new $\mathrm{Zn}$ (II) coordination polymers constructed from mixed ligands: $\mathrm{N}$ donor pridine ligands and bis(4-carboxylphenyl)phosphinic acid. J. Mol. Struct. 1180 (2019) 63-71.

12. Mo, J. P.; Bigdeli, F.; Yin, Y.; Zhu, C. Q.; Zhu, M.; Li, Y. S.; Li, X. H.; Xiao, H. P.; Morsali, A.; Ramazan, A.: Five new Cd(II) coordination polymers constructed from 4,4'(hydroxyphosphoryl)-dobenzoic acid and $\mathrm{N}$-donor pridine ligands. Polyhedron 158 (2019) 144-153.

13. Wu, L. F.; Wang, Z. X.; Xue, C. C.; Xiao, H. P.; Li, M. X.: Two $2 \mathrm{D}$ sliver complexes with a symmetric and noncentrosymmetric architecture controlled by the metal-ligand ratio. CrystEngComm 16 (2014) 5627-5632.

14. Li, L.; Wang, X.; Liang, J.; Huang, Y.-B.; Li, H.; Lin, Z.-J.; Cao, R.: Water-stable anionic metal-organic framework for highly selective separation of methane from natural gas and pyrolysis gas. ACS Appl. Mater. Interfaces 8 (2016) 9777-9781. 